\title{
Hydrocele in Pediatric Population
}

\author{
Ioannis Patoulias ${ }^{1}$, Efstathios Koutsogiannis², loannis Panopoulos², Panagiota Michou², \\ Thomas Feidantsis ${ }^{1}$, Dimitrios Patoulias ${ }^{3, *}$
}

\section{ABSTRACT}

Hydrocele is a collection of fluid within the tunica vaginalis. Based upon the etiology and the pathophysiology, it is divided into, the primary and secondary. The primary hydrocele includes the neonatal or the congenital, the communicating and the noncommunicating or the closed or the adult type. The secondary hydrocele can develop in the substrate of a pre-existing disease. After systematic and thorough systematic and thorough research of the relevant literature, we aim at describing all the aspects of this entity, with specific emphasis on the issues that remain unanswered from the scientific community.

\section{KEYWORDS}

congenital hydrocele; communicating hydrocele; closed or adult type hydrocele; secondary hydrocele; processus vaginalis; Nuck canal

\section{AUTHOR AFFILIATIONS}

${ }^{1}$ First Department of Pediatric Surgery, Aristotle University of Thessaloniki, General Hospital "G. Gennimatas", Thessaloniki, Greece

2 Department of Pediatrics, General Hospital "G. Gennimatas", Thessaloniki, Greece

${ }^{3}$ First Department of Internal Medicine, General Hospital "Hippokration", Thessaloniki, Greece

* Corresponding author: M. Alexandrou 3B, Pefka, Thessaloniki, Postal code 57010, Greece; e-mail: dipatoulias@gmail.com

Received: 26 September 2019

Accepted: 10 March 2020

Published online: 10 August 2020

Acta Medica (Hradec Králové) 2020; 63(2): 57-62

https://doi.org/10.14712/18059694.2020.17

(c) 2020 The Authors. This is an open-access article distributed under the terms of the Creative Commons Attribution License (http://creativecommons.org/licenses/by/4.0), which permits unrestricted use, distribution, and reproduction in any medium, provided the original author and source are credited. 


\section{ETIOLOGIC CLASSIFICATION}

Hydrocele is the collection of fluid within the tunica vaginalis of the testis. Based on the pathophysiological substrate, it is divided into primary and secondary. The primary hydrocele includes the neonatal or the congenital, the communicating and the non-communicating or closed type (1).

The secondary hydrocele can develop on the grounds of a pre-existing disease such as inflammation (epididymitis, epididymo-orchitis), testicular torsion or its embryonic appendages (appendiceal torsion), previous surgical intervention in the inguinal region or scrotum (e.g. varicocelectomy), hypoproteinemia due to a systemic disease and trauma or tumor of the intrascrotal structures. In thirdworld countries parasitic diseases (lymphatic filariasis, Wuchereria bancrofti etc.) are common causes of secondary hydrocele (2).

In the context of patent processus vaginalis pathology, the following disorders may occur: the processus vaginalis is almost obliterated from the level of the internal inguinal orifice. The rest of it fills with fluid which descends from the peritoneal cavity, so we have the neonatal or congenital hydrocele.

When the processus vaginalis remains obliterated on either side of a complete with fluid cavity, it is referred as a cystic hydrocele or a cyst of the spermatic cord. When the processus vaginalis is patent in its entire length, the result is the communicating hydrocele.

A particular entity is the abdomino-inguino-scrotal hydrocele. It is believed that the cause is the presence of a valve type obstruction of the processus vaginalis above the internal inguinal orifice. The fluid is collected up to the level of the internal inguinal orifice. In contrast to the large compliance of the scrotum, the inguinal canal is relatively small, due to its fibromuscular nature. The accumulated fluid within the processus vaginalis causes the development of high pressure at the internal inguinal orifice. However, this pressure overcomes the intra-abdominal one, then the hydrocele spreads intra-abdominally and so the abdominal part of the abdomino-inguino-scrotal hydrocele is formed. The collected fluid can be spread both intraperitoneally and retroperitoneally (3).

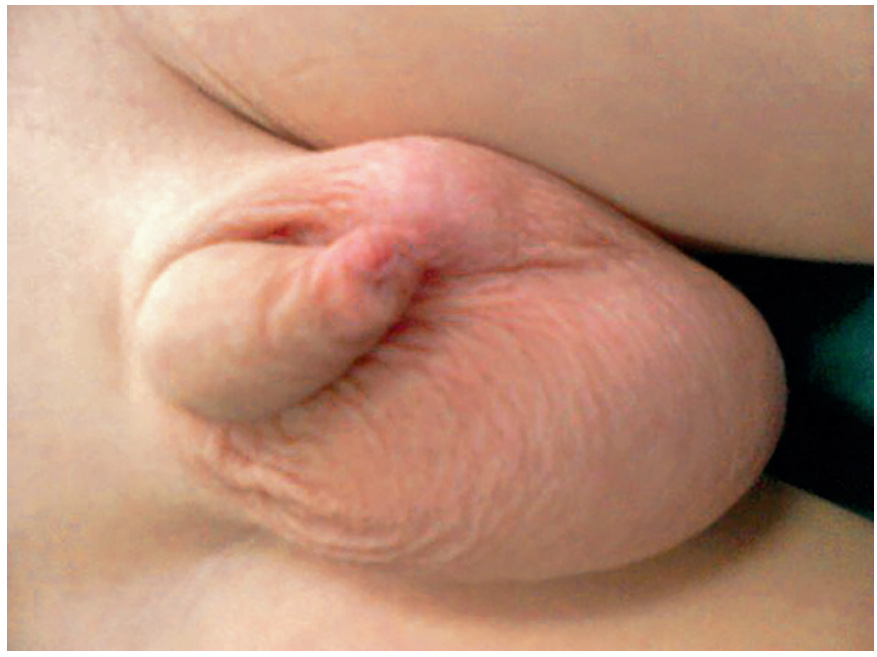

Fig. 1 Congenital hydrocele.
The non-communicating or closed type hydrocele most often manifests in prepubertal age. The mechanism of development remains unknown. It has been suspected that the cause could be a small communication between the processus vaginalis and the peritoneum that remains asymptomatic during early childhood and manifests later. However, with the surgical confirmation of an absence of a patent processus vaginalis, this hypothesis is disputed. Koutsoumis et al conducted a biochemical analysis of the fluid in 13 patients with a closed type hydrocele and found that it was serous fluid in all cases (4).

According to the latest postulation, the primary non-communicating hydrocele is caused by a disorder in the balance between the rate of production and reabsorption of the fluid from the tunica vaginalis epithelial cells. Therefore, it concerns either an increased rate of production, or a reduced rate or reabsorption of the collected fluid within the tunica vaginalis $(4,5)$.

\section{EMBRYOLOGY}

The processus vaginalis develops as a peritoneal protrusion during the 12th embryonic week. Gradually it exits from the internal inguinal orifice, it traverses the inguinal canal and in girls, it is inserted in the pubic tuberculum. In boys, it reaches the scrotum with its last part forming the two layers of the tunica vaginalis which surrounds in part the homolateral testis. The processus vaginalis takes part catalytically in the testicular descent from the lumbar region to the scrotum, like a "hydraulic" force.

After birth, the progressive obliteration of the processus vaginalis continues. Because the descent of the left testis is completed earlier, the obliteration of the right processus vaginalis is delayed (6). This fact explains the prevalence of right-side manifestation of the entities in the context of patent processus vaginalis (7). The processus is patent in $80-94 \%$ of newborn boys. Sachs proved that in ages ranging from 4 to 12 months, it remains patent in $57 \%$ of infants (8). Autopsy studies of adults showed that the processus vaginalis is patent in $5 \%(5-37 \%)$ of cases (9). In $80-88 \%$ of adult males the processus vaginalis is turned into a fiber chord after its obliteration. In females

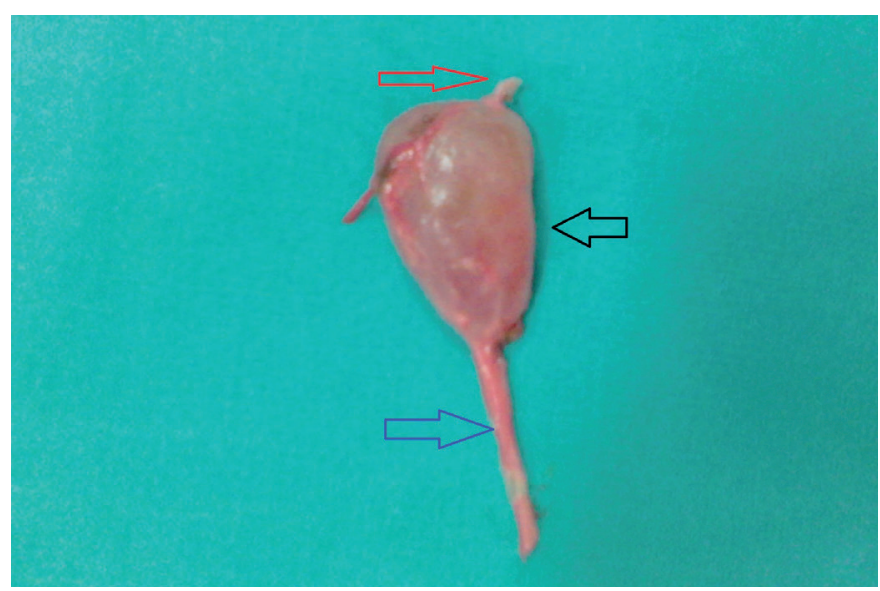

Fig. 2 Spermatic cord hydrocele. Notice the distal part of processus vaginalis (red arrow), the cyst (black arrow) and the proximal part of processus vaginalis (blue arrow). 
the corresponding structure is called the Nuck canal and it reaches the ipsilateral major labium.

The existence of a patent processus vaginalis is an essential but not catalytic factor for the development of an indirect inguinal hernia, because only $8-12 \%$ of adults develop indirect inguinal hernia (10).

Hutson et al. claim that the androgen-dependent action of the genitofemoral nerve through the secretion of a neuropeptide (calcitonin gene-related peptide, CGRP) is responsible for the physiologic obliteration of the processus vaginalis (11). Reduced secretion of CGRP prenatally causes disorders in the descent of the testis, while the reduced secretion antenatally leads to inguinal hernia or hydrocele. CGRP causes in vitro obliteration of the processus vaginalis in infants with inguinal hernia. Initially the peptide acts on the fibroblasts of the wall of the processus vaginalis, in which the presence of CGRP receptors have been documented. The mechanism however by which these changes occur in the mesothelium of the processus vaginalis that have been observed in vitro studies and the following obliteration have not been clarified yet $(11,12)$.

Tanye et al. believe that the failure of obliteration of the processus vaginalis is caused by the presence of smooth muscle fibers in its wall. This hypothesis is based on studies in which increased expression of different markers, such as actin and desmin in the processus vaginalis wall of patients with inguinal hernia or communicating hydrocele, were recorded (12). Based on these observations, the existence of smooth muscle fibers was proven in the wall of a patent processus vaginalis, in contrast to their absence from a normally obliterated one. However, the exact mechanism by which the smooth muscle fibers keep the processus patent is not completely understood.

The degeneration of myofibroblasts induces the apoptosis of the smooth muscle fibers and the mesothelium of the processus vaginalis, which leads to its obliteration. Therefore, disorders in this procedure cause disturbance in the normal obliteration of the processus vaginalis $(12,13)$.

It has been estimated that the quantity of smooth muscle fibers that remain on the wall of the processus vaginalis, correlates with the manifestation of either hydrocele(less), or inguinal hernia(more). In the above mechanism, the autonomic nervous system and activity of androgen disorders are involved, since these two factors are considered to affect the smooth muscle fibers. The androgens affect the fibers both directly and indirectly through the sympathetic nervous system, which has an androgen dependent activity. The sympathetic innervation plays an important trophic role for the smooth muscle fibers, increasing the intracellular cAMP through $\beta$-adrenergic receptors. Consequently, with the reduction of the activity of the sympathetic nervous system and the reactive increase in the parasympathetic activity, the smooth muscle cell apoptosis is induced (13).

Especially in females, due to a smaller number of sympathetic nerve fibers, the sympatholytic activity causes apoptosis of the smooth muscle fibers on the walls of the Nuck canal. Based on this analysis, the rarer manifestation of inguinal hernia in females might be explained $(13,14)$.

\section{CLINICAL MANIFESTATION AND EVOLUTION}

Due to a progressive obliteration of the processus vaginalis, the neonatal or congenital hydrocele is reversed on its own in $63-89 \%$ of cases until the age of $12-24$ months $(15,16)$. Indeed, this obliteration is completed within the first six months of life in $75 \%$ of cases (1).

If the processus vaginalis remains patent, however, then the communicating hernia develops. The communicating hydrocele usually presents during infancy and is bilateral. The increase and decrease of swelling are pathognomonic findings.

Hydrocele must be differentiated from the inguinal hernia especially when it expands to the scrotum. The cervix of the hydrocele is narrow at the level of the external inguinal orifice, in contrast to the inguinal hernia which expands into the inguinal canal. The content of the inguinal hernia can be repositioned, while the hydrocele one cannot be repositioned. The latter is difficult to assess in neonates and infants, especially if it concerns a hydrocele under pressure. In this case, a digital rectal examination is necessary to exclude an incarcerated inguinal hernia. During the effort of repositioning a hydrocele under pressure, the fluid can be directed internally through the external inguinal orifice, giving the impression of repositioning an incarcerated inguinal hernia. The hydrocele, however, is mobile and painless, while the inguinal hernia is fixed to the wall of the inguinal canal and is painful during palpation. Transillumination can contribute diagnostically, however an incarcerated air-filled intestinal helix can appear similarly to a hydrocele.

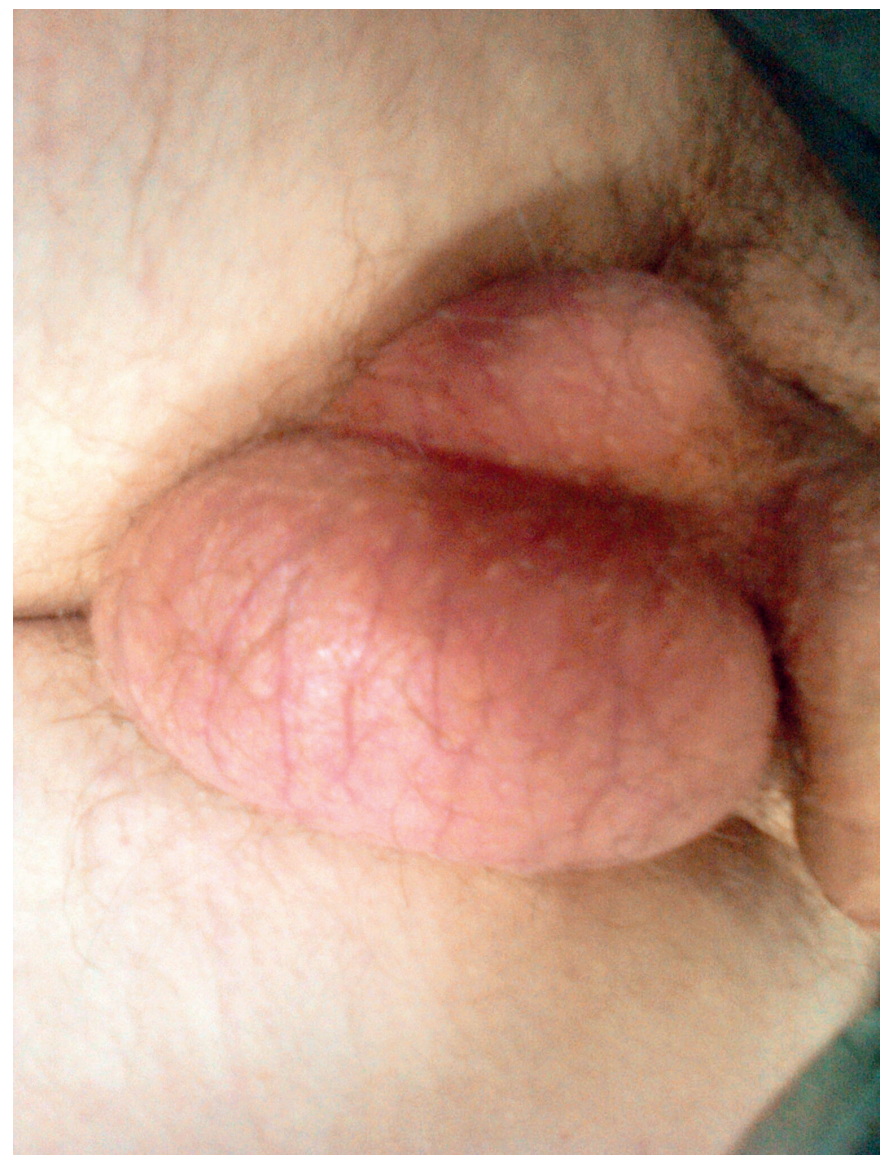

Fig. 3 Adult type hydrocele. 
The congenital or neonatal hydrocele should not worry the parents as in most cases it subsides within the first year of life. A periodic follow-up is required every 3-6 months during this period. Urgent evaluation may be required if it suddenly increases in size or pressure develops. If the hydrocele persists beyond first 1-2 years of life, then it possibly concerns a communicating one. Before the last 15 years, most pediatric surgeons were aggressive concerning the time of treatment, assuming it was potentially an inguinal hernia. Today a more conservative approach is followed (17). Besides that, the probability of inguinal hernia is less than $5 \%$ and without a reported episode of incarceration (16). A longer follow-up, however, is usually required.

In cases of delayed manifestation of hydrocele, Christensen et al. believe that the indication for surgical intervention is better to be set after a monitoring period of 6-9 months, during which there is a $75 \%$ chance of reversion (18). In spermatic cord hydrocele, surgical intervention is recommended, if it persists beyond the age of 18 months $(16,18)$.

In conclusion, the advised strategy is to avoid surgical procedure during the first 2 years of life, except for the following cases: a) the existence of inguinal hernia cannot be excluded, b) it concerns a large, symptomatic and under pressure hydrocele and c) it concerns a communicating hydrocele with frequent increase and decrease of its size, a fact that means that a great volume of fluid is being transferred between the peritoneal cavity and the processus vaginalis, so its width is large, suggesting a potential inguinal hernia. After the first two years of life the hydrocele must be surgically corrected if it does not tend to subside or if it manifests acutely, as at this age its reversion is extremely rare.

\section{PRINCIPLES OF MANAGEMENT}

The basis of the communicating hydrocele operative management is the high ligation of the processus vaginalis at the level of the internal inguinal orifice with creation of a fenestration in the homolateral tunica vaginalis. Reversion of the tunica vaginalis is not required (Bottle procedure) $(6,19)$. Reversion of the tunicae is indicated in a hydrocele under pressure as well, as in cases, where the tunica vaginalis is thickened, by fibrotic and with elements of inflammation $(6,19)$.

In tense neonatal hydrocele, in abdomino-scrotal hydrocele and in closed type hydrocele the procedure can be performed via scrotal approach with trans-scrotal incision. Tunica albuginea reversion, with or without tunica vaginalis excision, along with joining the tunicae- with suturesin the posterior surface of the testis, without spermatic cord compression (Lord method), is recommended (2-5).

The advantages of the scrotal approach include a better aesthetic result, reduction in operative time and no danger of damaging the ilioinguinal nerve (2-5).

\section{POSTOPERATIVE HYDROCELE}

The most characteristic example includes the hydrocele that is formed in the ipsilateral hemi-scrotum - usually the left - after varicocelectomy. Usually they develop within 2-22 months after surgery, although cases observed more than 6 years after surgery have been reported $(20,21)$. Possibly, in most cases, the post-surgical follow-up period is short, thus cases of hydrocele that develop late can be missed. It is not considered a relapse, since pre-surgically hydrocele does not exist. This entity affects $1-40 \%$ of males that have undergone varicocelectomy $(22,23)$. Etiologically it is believed that the cause is destruction or blockage of the lymphatic vessels, whose course is parallel to the internal spermatic vessels. It has been established that the hydrocele improves and reverses in $14-60 \%$ of cases, either due to development of collateral lymph circulation or regrowth of the blocked lymphatic vessels $(20,21)$.

In cases when the postoperative hydrocele persists beyond the first year after varicocelectomy, its surgi-
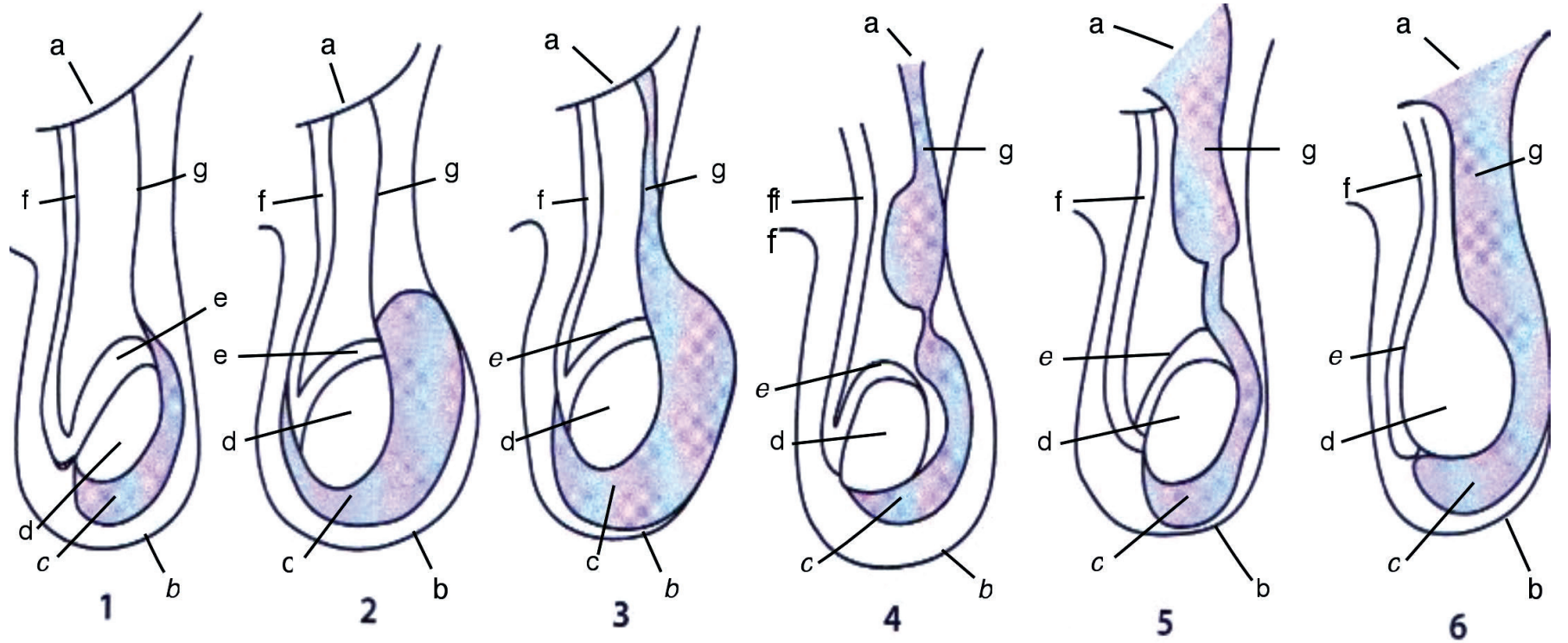

Fig. 4 1. Normal anatomy, 2. Adult type hydrocele, 3. Communicating hydrocele, 4. Spermatic cord hydrocele, 5. Inguinal hernia, 6. Inguinoscrotal hernia; a. peritoneal cavity, b. scrotum, c. tunica vaginalis, d. testis, e. epididymis, f. spermatic duct, g. processus vaginalis. 
cal management is indicated. Initially, aspiration under aseptic conditions is recommended and, if it relapses, hydrocelectomy by scrotal approach is required. It should be noted that the possibility of relapse is much greater than the documented in other cases of hydrocele. Esposito et al. in their small series observed that 2 out of 6 cases with postoperative hydrocele in the context of varicocelectomy finally relapsed (22).

\section{HYDROCELE RECURRENCE}

Recurrence usually develops after the treatment of inguinal hernia or hydrocele of the homolateral inguinal region. Commonly, this occurs several months postoperatively. Morecroft et al. treated 556 males with hydrocele or inguinal hernia and observed that 8 out of $556(1.4 \%)$ developed hydrocele postoperatively (23). Ein et al. treated 5,343 males with hydrocele or inguinal hernia and postoperative hydrocele developed only in 2 patients $(0.038 \%)$ (24). Davies et al. found that the hydrocele recurrence was greater $(11 \%)$ when the male patients that had undergone surgery for hydrocele or inguinal hernia weighted less than 3 kilograms (25). In the contrary, Moss et al. demonstrated that only 2 out of 328 neonates $(0.6 \%)$ experienced hydrocele recurrence (26).

There is no algorithm concerning the surgical strategy that should be followed for this complication $(21,27)$. The first step is the observational approach for at least 6 months. The next step is the trans-scrotal paracentesis and the absorption of the fluid with the use of a needle under aseptic conditions and local anesthesia, in cases when the hydrocele persists, or the volume of the collected fluid increases, or local discomfort appears. A contraindication for this procedure is the existence of homolateral inguinal hernia or communicating hydrocele.

Surgical management is advised if the fluid aspiration - which can be repeated up to 5 times - does not solve the problem. Surgical intervention can be performed either via scrotal or inguinal incision. The majority of pediatric surgeons prefer the inguinal approach, as this allows the exploration and correction of a potential inguinal hernia or a communicating hydrocele.

The inguinal approach is also advised in those cases, in which the first operation was performed trans-scrotally, because the anatomy and the potential presence of inguinal pathology were not thoroughly assessed.

\section{NUCK'S HYDROCELE}

Nuck's hydrocele concerns a communicating hydrocele in females resulting from the persistence of a patent Nuck's canal, the equivalent of the processus vaginalis in males. Nuck's canal was named after the Dutch Anton Nuck, who first described this anatomic entity (28). This peritoneal protrusion accompanies the round ovarian ligament in its extra-abdominal course. It passes through the inguinal canal and attaches to the ipsilateral pubic tuberculum. The wall of this peritoneal protrusion is comprised of mesothelial cells with single or multi-layered cuboidal or cylindrical epithelium internally, which are surrounded by a thick fibrotic connective tissue, which is in turn traversed by bundles of smooth muscle fibers.

It is possible for the Nuck's canal to obliterate, resulting in fluid accumulation within the canal due to an imbalance between the production rate from the mesothelial cells and the rate of its absorption.

Nuck's hydrocele can be classified into 4 types (29):

- the equivalent to the spermatic cord cyst in males,

- the equivalent to communicating hydrocele in males,

- the "hour glass" type or the type of two-space hydrocele. In this type, two cavities are observed, one peripheral, closed type and one central that communicates with the peritoneal cavity through the patent Nuck's canal,

- the equivalent to the non-communicating or closed type hydrocele in males.

It is a rare entity, as relevant literature mainly consists of case reports or small case studies. In general, Nuck's hydrocele is 8 times rarer than the communicating hydrocele in males $(28,29)$. It is characterized by painless and non-reversible swelling, usually mobile, with well-defined boundaries, located in the right inguinal region that can expand to the ipsilateral major labium of the vagina. Swelling can range from 2.3 to $5.6 \mathrm{~cm}(28)$. The physician must differentiate it from an incarcerated inguinal hernia or an incarcerated femoral hernia, especially from one which contains ovary or part of the small intestine or omentum or a lipoma, the soft tissue tumors of the inguinal area and the inguinal region lymphadenopathy.

It is worth highlighting that $98.7 \%$ of cases of swelling of the inguinal region in females correspond to an inguinal hernia and only $0.76 \%$ to Nuck's hydrocele (28).

Diagnosis is confirmed by ultrasonography. Major findings are the hypo-echoic or anechoic content, the single cavity or multiple cavities with thin septa and the depiction of the "comma sign" (29). Rarely, magnetic resonance imaging may be required.

Treatment of choice is the ligation of the Nuck's canal at the level of internal inguinal orifice - as long as it is patent - and its removal down to its peripheral attachment to the homolateral pubic tuberculum (29).

\section{CONFLICT OF INTEREST}

None declared.

\section{REFERENCES}

1. Dave J. Cause and management of hydrocele: a review article. Indian J of Applied Research 2015; 5: 117-8.

2. DeVries $C$. The role of urologist in the treatment and elimination of lymphatic filariasis worldwide BJU Int 2002; 89: 37-43.

3. Belman $A B$. Abdominoscrotal hydrocele in infancy: a review and presentation of the scrotal approach for correction. J Urol 2001; 165: 225-7.

4. Koutsoumis G, Patoulias I, Kaselas C. Primary new-onset hydroceles presenting in late childhood and pre-adolescent patients resemble the adult type hydrocele pathology. J Pediatr Surg 2014; 49: 1656-8.

5. Wilson JM, Aaronson DS, Schrader R, Baskin LS. Hydrocele in the pediatric patient: inguinal or scrotal approach? J Urol 2008; 180: 1724-8.

6. Holcomb GW, Murhy JP, Ostlie DJ: Ashcraft's Pediatric Surgery. 6th edition. Elsevier, Saunders Co: New York 2014, Chapter 50, pp 679-88. 
7. Harper RG, Garcia A, Sia C. Inguinal hernia: A common problem of premature infants weighing 1,000 grams or less at birth. Pediatrics 1975; 56: 112-5.

8. Chevrel IP. Surgery of the abdominal wall. Springer Verlag: Berlin 1987.

9. Gray SW, Skandalakis JE. Embryology for surgeons. 2nd ed. Baltimore: Williams \& Wilkins 1994, pp 540-93.

10. Celik A, Ergón O, Arda MS, et al. The incidence of inguinal complications after ventriculoperitoneal shunt for hydrocephalus. Childs Nerv Syst 2005; 21: 44-7.

11. Hutson JM, Hasthorpe S, Heyns CF. Anatomical and functional aspects of testicular descent and cryptorchidism. Endocr Rev 1997; 18: 259-80.

12. Hutson JM, Albano FR, Paxton $G$, et al. In vitro fusion of human inguinal hernia with associated epithelial transformation. Cells Tissues Organs 2000; 166: 249-58.

13. Tanyel FC, Okur HD. Autonomic nervous system appears to play a role in obliteration of processus vaginalis. Hernia 2004; 8: 149-54.

14. Hosgor M, Karaca I, Ozer E, et al. The role of smooth muscle cell differentiation in the mechanism of obliteration of processus vaginalis. J Pediatr Surg 2004; 39: 1018-23.

15. Naji $\mathrm{H}$, Ingolfsson I, Isacson D, et al. Decision making in the management of hydroceles in infants and children. Eur J Pediatr 2012; 171: 807-10.

16. Koski M, Makari J, Adams M. Infant communicating hydroceles-do they need immediate repair or might some clinically resolve? J Pediatr Surg 2010; 45: 590-3.

17. Antonoff MB, Kreykes NS, Saltzman DA et al. American Academy of Pediatrics Section on surgery hernia survey revisited. J Pediatr Surg 2005; 40: 1009-14.

18. Christensen T, Cartwright PC, Devries C, Snow BW. New onset of hydroceles in boys over 1 year of age. Int J Urol 2006; 13: 1425-7.
19. Andrews EW. The "Bottle Operation" method for the radical cure of hydrocele. Ann Surg 1907; 46: 915-8.

20. Chang Y-T, Lee J-Y, Wang J-Y, et al. Hydrocele of the spermatic cord in infants and children: Its particular characteristics. Urology 2010; 76 : $82-6$

21. Glick PL, Boulanger SC. Inguinal hernias and hydroceles. In: Grosfeld JL, O’Neill JA Jr, Fonkalsrud EW, Coran AG, (eds.), Pediatric surgery. 6th edition. Vol. 2. Philadelphia: Mosby 2006: 1172-92, Chapter 30 / Recurrent Hernia, Hydrocele, and Varicocele, p 485.

22. Esposito C, Montupet P. Laparoscopic treatment of recurrent inguinal hernia in children. Pediatr Surg Int 1998; 14: 182-4.

23. Morecroft JA, Stringer MD, Homes SJK, et al. Follow-up after inguinal herniotomy or surgery for hydrocele in boys. Br J Surg 1993; 80: 1613-4.

24. Ein SH, Njere I, Ein A. Six thousand three hundred sixty-one pediatric inguinal hernias: a 35-year review. J Ped Surg 2006; 41: 980-6.

25. Davies BW, Fraser N, Najmaldin AS, et al. A prospective study of neonatal inguinal herniotomy: the problem of the postoperative hydrocele. Pediatr Surg Int 2003; 19: 68-70.

26. Moss RL, Hatch EI. Inguinal hernia repair in early infancy. Am J Surg 1991; 161: 596-9.

27. Lloyd DA. Inguinal and femoral hernia. In: Ziegler MM, Azizkhan RG, Weber TR, (eds.), Operative pediatric surgery. New York: McGaw-Hill 2003, p 543.

28. Akkoyun I, Kucukosmanoglu I, Yalinkilinc E. Cyst of the canal of Nuck in pediatric patients. N Am J Med Sci 2013; 5: 353-6.

29. Stickel WH, Manner M: Female hydrocele (cyst of the canal of Nuck): sonographic appearance of a rare and little-known disorder. J Ultrasound Med 2004; 23: 429-32. 